Олег ПЕТРАШЕВСКИЙ, ${ }^{1}$ Александр АЛЕКСЕЕНКО ${ }^{2}$, Владимир ДАНИЛЕВСКИЙ

\title{
АДЕКВАТНОСТЬ КРИТЕРИЕВ РИСКА И ОШИБОК ДИАГНОСТИРОВАНИЯ ТЕХНИЧЕСКОГО СОСТОЯНИЯ ТРАНСПОРТНЫХ СИСТЕМ
}

В статье показана адекватность вероятностных характеристик диагностирования объекта транспортной системы, включая ошибки 1-го и 2-го рода, и количественные меры рисков оценки технического состояния объектов диагностики. При анализе нестандартных (проблемных) ситуаций транспортных процессов, в которых наблюдается появление совместных событий, таких как отказ уже эксплуатируемого объекта диагностирования и транспортного происшествия, вызванного этим отказом, оказывается, что следует учитывать количественную меру риска диагностирования, как комплексную характеристику, принимая во внимание все риски, возникающие в процессе диагностического контроля объектов.

Следующим шагом в исследовании организационно-технических рисков научноисследовательских проектов в области транспорта является переход к нечетким моделям взаимозависимости категорий, участвующих в рассматриваемых процессах. Данная задача является также непростой - она требует экспертной оценки субъектов, специалистов в области транспортных процессов и технологий, участвующих в научно-исследовательских проектах транспортной тематики, начиная с постановки проблем, решения их, реализации принятых решений и сопровождения полученных результатов в эксплуатационных условиях.

Подтверждение адекватности критериев риска и ошибок диагностирования технического состояния транспортных систем позволяет с достаточной достоверностью идентифицировать и предметно определить сущность функций принадлежностей итогового показателя критерия риска. Данный факт получен при допущении совпадения координатных плоскостей функций принадлежности, совпадающих друг с другом а именно пределами их изменения от 0 до 1 . Основные субъективные оценки итоговой функции принадлежности критерия риска при этом превращаются в задачу объективного характера.

Ключевые слова:Транспортная система, техническая диагностика, риск, нечеткая логика, функция принадлежности, техническое состояние объекта.

\footnotetext{
${ }^{1}$ Олег Петрашевский - др технических наук, проф., кафедра аэропортов Национального транспортного университета, Украина, Киев 01010, ул. Суворова, 1; Тел.: +38 (044) 280-70-73; e-mail: gip65n@gmail.com (corresponding author).

2 Александр Алексеенко - мгр, аспирант кафедры аэропортов Национального транспортного университета, Украина.

3 Владимир Данилевский - мгр, аспирант кафедры аэропортов Национального транспортного университета, Украина
} 


\section{1. ВВЕДЕНИЕ}

После получения диагностической информации о состоянии объекта диагностирования (ОД) может быть принята одна из гипотез: $\Gamma_{1}-$ ОД исправен и $\Gamma_{2}$

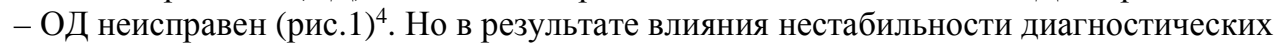
параметров ОД, недостаточности точностных характеристик функционирования диагностических комплексов (ДК), помех со стороны среды диагностирования появляется возможность ошибочных решений процесса диагностирования: часть работоспособных ОД признается неработоспособной, а часть неисправных объектов принимается исправными и допускаются к эксплуатации по назначению. Данный факт показан на рис.1, где:

- $\Gamma_{1}=\left\{\mathrm{H}_{11}, \mathrm{H}_{10}\right\}$, гипотеза $\mathrm{H}_{11}$ состоит в том, что ОД является исправным и это соответствует истинному техническому состоянию ОД, гипотеза $\mathrm{H}_{10}$ заключается в том, что ОД является неисправным при фактической исправности объекта контроля;

$\Gamma 2=\left\{\mathrm{H}_{00}, \mathrm{H}_{01}\right\}$, гипотеза $\mathrm{H}_{00}$ заключается в том, что ОД является неисправным и это соответствует фактическому техническому состоянию (ТС) объекта диагностирования, гипотеза $\mathrm{H}_{01}$ - ОД является исправным, что не соответствует истинному ТС ОД.

Гипотезы $\mathrm{H}_{11}$ и $\mathrm{H}_{00}$ являются правильными, а $\mathrm{H}_{10}$ и $\mathrm{H}_{01}$ - неправильными. Из сказанного следует сделать вывод: правильные решения не представляют интереса с точки зрения анализа организационно-технических рисков научно-технических и научно-исследовательских проектов, согласно которых исследуются, разрабатываются, реализуются, внедряются и эксплуатируются диагностические комплексы транспортных систем. Объектом исследований должны стать гипотезы $\mathrm{H}_{10}$ и $\mathrm{H}_{01}$ (рис.2).

\section{2. ОСНОВНАЯ ЧАСТЬ}

Решение о неисправности исправного ОД приводит к повторному ремонту объекта контроля, то есть дополнительным материальным затратам, и так может быть много раз. Гипотеза $\mathrm{H}_{01}$ при которой принимается решение об исправности фактически неисправного объекта диагностирования является наиболее отрицательным выводом. Латентно неисправный ОД, установленный на транспортное средство, несет в себе повышенный риск внезапного отказа и, как следствие, реальную возможность возникновения транспортного происшествия, иногда с катастрофическими последствиями.

\footnotetext{
4 Данилевський В.В. Класифікація організаційно-технічних ризиків на станах виконання науководослідних проектів /В.В.Данилевський//Збірник доповідей 14-ої Міжнародної науково-практичної конференції «Ринок послуг комплексних транспортних систем та прикладні проблеми логістики».К.:НTУ, 2012.-C.170-174.
} 


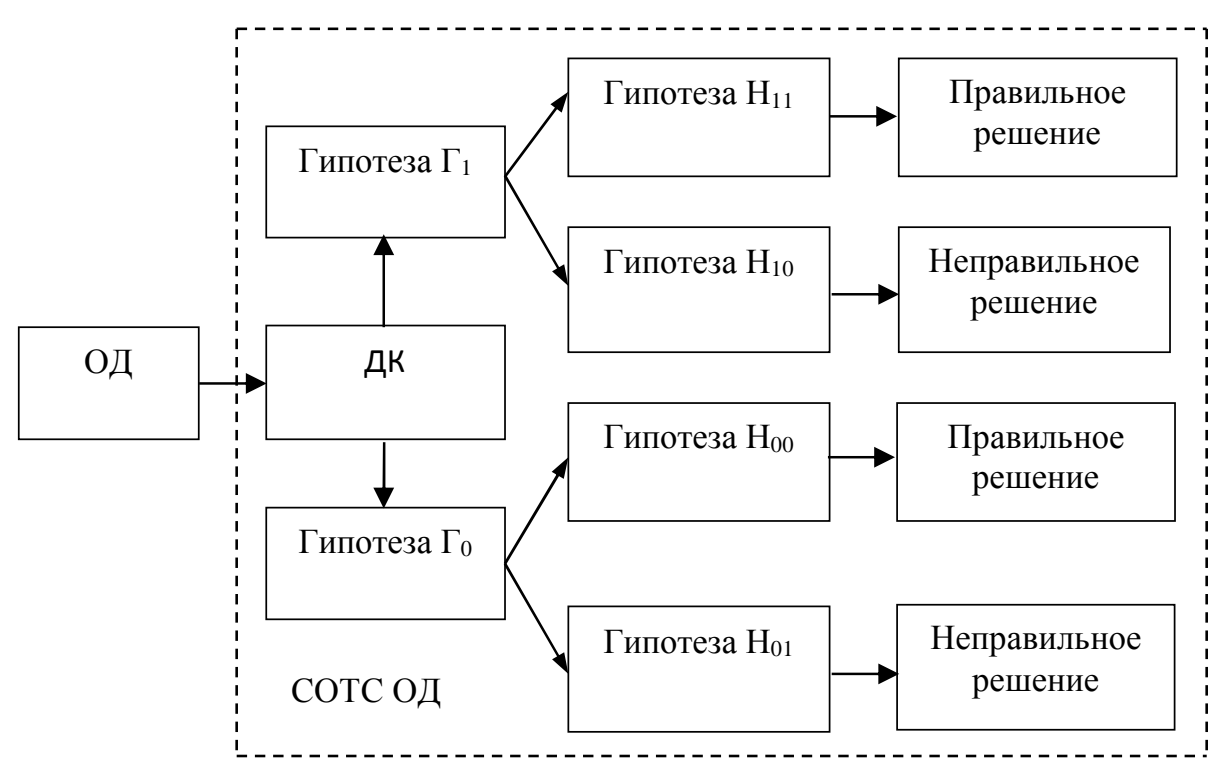

Рис.1. Процедура принятия решения в процессе диагностики ОД на диагностическом комплексе.

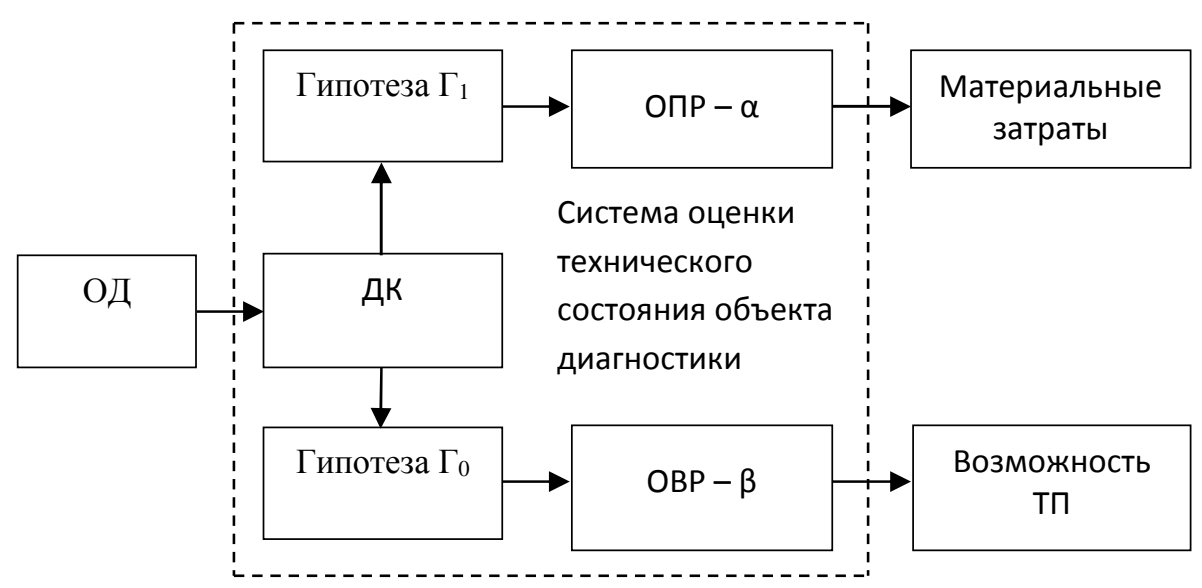

Рис.2. Ошибки 1-го и 2-го рода, приводящие к возможным негативным последствиям в процессе эксплуатации ОД.

Ошибки определения значения диагностического параметра в процессе контроля ОД на диагностическом комплексе называются: при гипотезе $\mathrm{H}_{10}$ - ошибка 1-го рода (ОПР), обозначается $\alpha$, а при гипотезе $\mathrm{H}_{01}$ - ошибка 2-го рода (OBР), 
обозначается $-\beta$ (рис. 2$)^{5}$. Следовательно, можно заключить, что организационнотехнические (ОТ) риски, возникающие в процессе выполнения научноисследовательских проектов реализующих разработку и внедрение новых транспортных систем (ТС) и их ДК - а сейчас именно так ставится вопрос, так как практически на всех видах транспорта интенсивно используется эксплуатация ТС по состоянию с контролем параметров - адекватны рискам, которые формируются в системах оценки технического состояния ОД на диагностических комплексах.

В первую очередь, данные риски воплощаются в ошибках $\alpha$ и $\beta$. Риск, возникающий при признании исправного ОД неисправным - Rин, адекватен ошибке 1-го рода $\alpha$, а Rни - ошибке 2-го рода $\beta$ (рис.3).

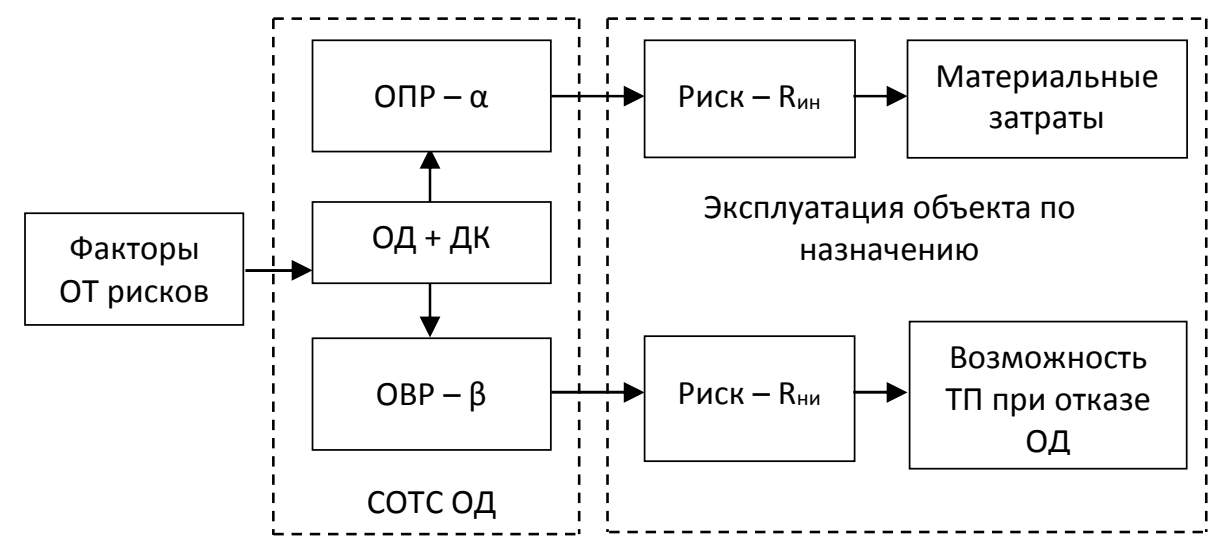

Рис.3. Адекватность ошибок 1-го и 2-го рода процесса диагностики количественным мерам организационно-технических рисков оценки технического состояния объекта диагностики.

Размерности ошибок и рисков совпадают - первые имеют функцию распределения, как вероятностная характеристика, на интервале [0,1], вторые функции принадлежности, как нечеткие множества, на том же интервале. Многие исследователи, работающие в области рискологии, рассматривают разного рода риски как вероятности, хотя строго говоря, риски являются возможностными характеристиками категории бытия и подчиняются зависимостям в рамках математического аппарата нечеткой логики ${ }^{6}$.

Вместе с тем, при анализе нестандартных (проблемных) ситуаций транспортных процессов, при которых наблюдается появление совместных событий - отказа уже эксплуатируемого ОД и транспортного происшествия, вызванного этим отказом, выявляется тот факт, что следует учитывать количественную меру риска

\footnotetext{
5 Давыдов П.С. Техническая диагностика радиоэлектронных устройств и систем.- М.: Радио и связь, 1988. - 256c.

${ }^{6}$ Петрашевский О.Л. Нечеткие теоретико-множественные модели процесса обеспечения безопасности движения автомобильного транспорта / О.Л. Петрашевский, И.П. Гамеляк, А.В. Алексеенко // Проблеми транспорту. Збірникнауковихпраць:Випуск 8.-К.:НТУ, 2011.- С.30-41.
} 
диагностирования как комплексную характеристику, принимая во внимание все риски, возникающие в процессе диагностического контроля объектов. И это следует делать по каждому из контролируемых параметров. Поэтому на рис.4 представлена структура формирования риска диагностики $-\mathrm{R}_{\text {д }}$, начиная с нижних уровней.

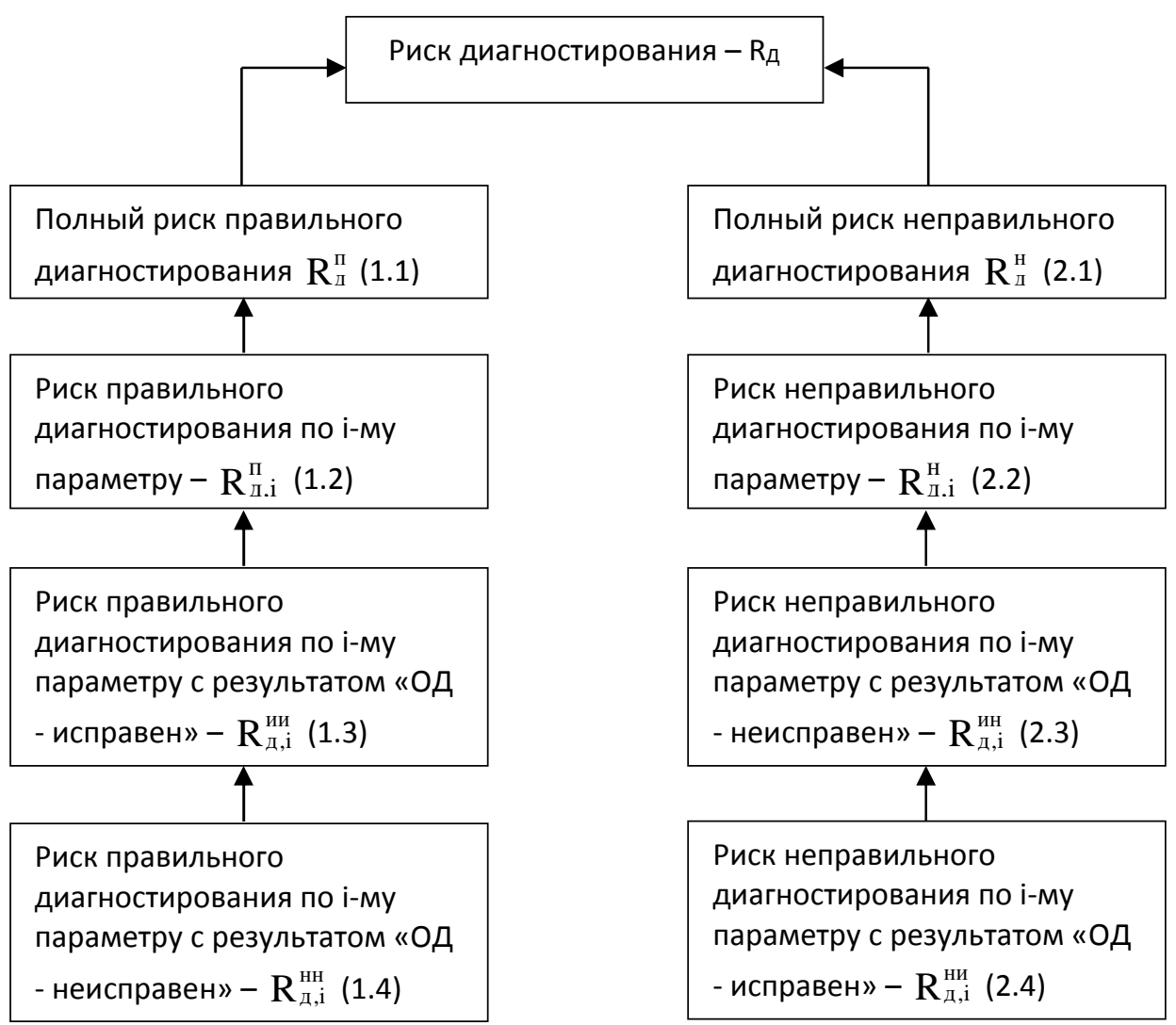

Рис.4. Структура формирования риска диагностики ОД на ДК.

Зная вероятности появления событий $\mathrm{P}\left(\mathrm{H}_{11}\right), \mathrm{P}\left(\mathrm{H}_{00}\right), \mathrm{P}\left(\mathrm{H}_{10}\right), \mathrm{P}\left(\mathrm{H}_{01}\right)$, как реализаций соответствующих гипотез, можно определить всю номенклатуру рисков. Правда следует принять следующие допущения:

- диагностические параметры, общим количеством $\mathrm{i}=\overline{1, \mathrm{n}}$, являются независимыми друг от друга переменными и обладают каждый своими допусками, что фактически, в реальности не так;

- аналитические выражения для определения вероятностей гипотез, которые в практике также взаимосвязаны, весьма сложны, поэтому есть смысл своевременно перейти на использование математического аппарата нечетной логики (теории возможностей Л.Заде) и субъективных экспертных баз знаний; 
- диагностические комплексы вносят собственные погрешности в измеряемые параметры ОД, но всегда находятся в исправном состоянии, в действительности же необнаруженный отказ ДК приводит к тому, что он может браковать все контролируемые объекты диагностики.

С учетом вышеприведенного формулы для вычисления рисков снизу вверх - это показывает двойная нумерация на рис.4 - имеют следующий вид:
(1.4) $\underset{\mathrm{R}_{\text {д, } \mathrm{i}}^{\mathrm{HH}}}{=}={ }_{1}-\mathrm{Pi}\left(\mathrm{H}_{00}\right)$;
(2.4) $\underset{\mathrm{R} \text { д, } \mathrm{i}}{\text { ни }}=\beta_{\mathrm{i}}=1-\frac{\mathrm{P}_{\mathrm{i}}\left(\mathrm{H}_{11}\right)}{\mathrm{P}_{\mathrm{i}}\left(\mathrm{H}_{11}\right)+\mathrm{P}_{\mathrm{i}}\left(\mathrm{H}_{01}\right)}$;
(1.3) $\underset{\text { ид, }}{\text { ии }}={ }_{1}-{ }_{\mathrm{Pi}}\left(\mathrm{H}_{11}\right)$;
(2.3) $\underset{\mathrm{R}_{д, i}}{\text { ин }}=\alpha_{\mathrm{i}}=1-\frac{\mathrm{P}_{\mathrm{i}}\left(\mathrm{H}_{00}\right)}{\mathrm{P}_{\mathrm{i}}\left(\mathrm{H}_{00}\right)+\mathrm{P}_{\mathrm{i}}\left(\mathrm{H}_{10}\right)}$;

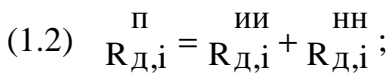

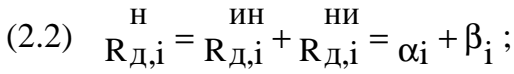
(1.1) $\mathrm{R}_{\text {д }}^{\Pi}=\sum_{\mathrm{i}=1}^{\mathrm{n}} \mathrm{R}_{\text {д, }}^{\Pi}$;
$\stackrel{\mathrm{R}_{\text {д }}}{\mathrm{H}}=\sum_{\mathrm{i}=1}^{\mathrm{n}} \underset{\mathrm{R}_{\text {д, }}}{\mathrm{H}}$.

В итоге: $\mathrm{R}_{д}=\mathrm{R}_{\text {Д }}^{\Pi} \cdot \mathrm{R}_{\text {д }}^{\mathrm{H}}$.

\section{3. ВЫВОДЫ}

Таким образом, показана адекватность вероятностных характеристик диагностирования ОД транспортной системы, включая ошибки 1-го и 2-го рода, и количественные меры рисков оценки технического состояния объектов диагностики. Следующим шагом в исследовании организационно-технических рисков научно-исследовательских проектов в области транспорта будет переход к нечетким моделям взаимозависимости категорий участвующих в рассматриваемых процессах.

Данная задача является также непростой - она требует экспертной оценки субъектов, специалистов в области транспортных процессов и технологий, участвующих в научно-исследовательских проектах транспортной тематики, начиная с постановки проблем, решения их, реализации принятых решений и сопровождения полученных результатов в эксплуатационных условиях. 


\section{СПИСОК ЛИТЕРАТУРЫ}

[1] Данилевський В.В. Класифікація організаційно-технічних ризиків на станах виконання науково-дослідних проектів /В.В.Данилевський//Збірник доповідей 14-ої Міжнародної науково-практичної конференції «Ринок послуг комплексних транспортних систем та прикладні проблеми логістики».- К.:НТУ, 2012.-С.170-174.

[2] Давыдов П.С. Техническая диагностика радиоэлектронных устройств и систем.- М.: Радио и связь, 1988. - 256с.

[3] Петрашевский О.Л. Нечеткие теоретико-множественные модели процесса обеспечения безопасности движения автомобильного транспорта / О.Л. Петрашевский, И.П. Гамеляк, А.В. Алексеенко // Проблеми транспорту. Збірникнауковихпраць:Випуск 8.K.:НTУ, 2011.- C.30-41.

[4] http://matlab.exponenta.ru/fuzzylogic/

\section{ADEQUACY CRITERION OF RISK AND ERRORS TECHNICAL DIAGNOSTIC CONDITION OF TRANSPORT SYSTEMS}

The article shows the value of probabilistic characteristics of diagnosing object diagnosing transport system, including errors of the 1st and 2nd kind, and quantitative measures of risk assessment of technical condition of diagnosis. In the analysis of non-standard (problematic) situation in the transport processes in which there is the emergence of common events, such as the refusal of the object of diagnosing, already being in operation, and the case of transport caused by the refusal to disclose the fact that one should take into account the quantitative risk measures diagnosing the complex characteristics, taking into account all the risks arising in the course of the diagnostic control objects.

The next step in the study of organizational and technical risks of research projects in the field of transport will go to an imprecise model of interdependence categories involved in these processes. This task is also difficult - it requires professional judgment of entities, experts in the processes and technologies involved in research projects within transport, from the formulation of the problems and their solutions, implementation of decisions implementing and tracking results in the operating conditions.

The confirmation of the adequacy of the risk criteria and error diagnostics of the technical condition of transportation systems allows, with sufficient reliability, to identify and objectively determine the substance of the final membership rate risk criteria. This statement is the result, obtained assuming the intersection of the coordinates that overlap with each other within changes from 0 to 1 . The basic assessments of final function risk criterion transform the objective problem.

Keywords: Transport system, technical diagnostics, risk, fuzzy logic, membership function, technical state of an object

\section{DOI: 10.7862/rz.2015.mmr.59}

Tekst złożono w redakcji: lipiec 2015

Przyjęto do druku: listopad 2015 